\title{
Measuring Irrationality in Financial Markets
}

\author{
Tobias Schädler \\ Universidad Nacional de Educación a Distancia (UNED), \\ Madrid,Spain
}

\begin{abstract}
This paper presents the measurement of irrationality contained in the continuous pricing of individual stocks. Irrationality is used to extend the concept of historical volatility by decomposing historical stock quotes into frequencies via Fourier transformation. The analysis in the frequency domain enables clustering of the contributions of short and long-term fluctuations to the overall price changes. With the resulting ratio it is possible to rank stocks within an index according to their specific fluctuation profile. The analysis is performed on daily stock quotes over a period of 20 years (1997-01-02 until 2016-12-30). Although the analysis presented here focuses on the stock market, the concept of irrationality is transferable to other financial markets as for bonds, housing prices or derivatives as well as to different time periods.
\end{abstract}

Keywords: irrationality, volatility, risk, speculative behavior, Fourier transform

\section{INTRODUCTION}

Mandelbrot proved that price movements of stock markets do not always follow a Gaussian distribution, what he describes as mild randomness. Instead historical stock quotes should be better described by a stable Paretian process to account for wild randomness [1]. The wild behavior of stock markets can be investigated with the use of Fourier techniques. The spectral density being proportional to $1 / f^{\alpha}$ and $\alpha=2$ in the frequency domain proves for mild randomness known as red noise or random walk. In this case the Gaussian distribution would hold, and the market is seen as efficient. Mild behavior of the market with thin tailed distributions therefore relates to the efficient market hypothesis (EMH) as described by Fama $[2,3] . \alpha<2$ proves for wilder randomness and may be described with the concept of irrational exuberance [4] which is better suited to explain fat tails. Furthermore, irrational behavior and therefore unexplained price movements by the EMH may be induced by the divergence of value and price as illustrated by Summers [5] as well as Shiller [6] and in a more recent study by Appel and Grabinski [7].

The purpose of this publication is to develop an applicable method to quantify the extent of irrationality in the valuation of the share prices of corporates. The resulting ratio is consciously named irrationality to express that it does not only include speculative behavior of market participants but also the behavior of the corporate management including its decisions. Other influences such as political decisions or interest rate decisions by central banks influence the overall market and are not company-specific. Therefore, stocks within an index should be comparable. The general idea is to transfer the time series into the spectral domain, where it is possible to measure the influence of specified frequency ranges with longer frequencies from three months to one year to short ones ranging from ten days to three months. The reason to exclude the frequencies below ten days is that with only one data point per day for the calculation, this range equals white noise and is further based on unreliable data in this range. This could lead to signals which are nothing else than data errors. 
Even though there is irrational behavior in the timespan from the tick basis to three months, it is not possible to separate adjustments to new information from components of irrationality like speculation. Long-term changes of over one year reflect actual changes in the macroeconomic environment as well as strategic decisions made by the board. So, the timespan between three month and twelve months was taken as the closest representation for irrationality.

Irrationality in this sense is neither good or bad per se, it is the part not explained by rational behavior as proposed by the EMH. It is also not indicating under- or overvaluation of the asset as neither fundamental values nor discounted cash flows are considered. Therefore, stock prices even though they adjust to new information on the short-term do not need to reflect the actual future economic benefits of the stock.

For the spectral estimation of functions or time series a broad literature body exists which is still extending. The mathematical details leading to the spectral estimation method used in this paper are given in the next chapter where the presented method to stock prices will be applied as well.

In the third chapter the results for stocks within an index as well as between indices are discussed. Therefore, a ratio of low frequencies to high frequencies closely linked to the concept of empirical probability is build. It determines the amount of irrationality in contrast to the inherent variance of the specific stock for adjusting to new information. Please note that this is a relative measure. It can be transformed to fit into analyses based on volatility or beta if needed. To get the real proportions of the power frequency bands, one may divide the sum of the irrational band by the short-term basis, which will stretch the results but keep the ranking.

The fourth and last chapter summarizes the results and gives hints for further research. The main result of this analysis is that by applying Fourier techniques, the strength of different periodic components within financial time series can be used to measure and classify risk within an index.

\section{THE FOURIER TRANSFORMATION}

The Fourier transformation is an over 200-year-old tool mostly applied to analyze frequencies in a signal (spectral analysis) or to solve an arbitrary set of linear partial differential equations. First, the Fourier series is introduced.

Any periodic function $f(t)$ can be written as a series of harmonic functions:

$$
f(t)=\sum_{k=0}^{\infty} a_{k} \cos (k \cdot \omega t)+b_{k} \sin (k \cdot \omega t)
$$

Here a period of $T$ was assumed so that $\omega=2 \pi / T k \in \mathbb{N}_{0}$. The coefficients $a_{k}$ and $b_{k}$ are determined by

$$
a_{k}=\frac{2}{T} \int_{0}^{T} d t f(t) \cdot \cos (k \cdot \omega t) \quad \text { and } \quad b_{k}=\frac{2}{T} \int_{0}^{T} d t f(t) \cdot \sin (k \cdot \omega t)
$$

A proof of Eq. (2) is performed by inserting $f(t)$ of Eq. (1) into Eq. (2) and performing the integration. A Fourier series exists only if the integrals of Eq. (2) exist. 
The interpretation of a Fourier series evaluates $a_{k}$ and $b_{k}$ which represent the strength of the frequency $k \omega$. Eq. (1) can also be used as an approximation for a periodic function e.g. in forecasting. Then $a_{k}$ and $b_{k}$ are considered fit-parameters. This is analogous to a Taylor series up to a certain power.

A series of stock prices is most likely not periodic. Analyzing the frequencies of stock prices for e.g. 20 years, one may assume that stock prices have a period of 20 years.

Instead of having discrete frequencies, also continuous frequencies can be applied. $a_{k}$ or $b_{k}$ are then becoming a function rather than a set of discrete parameters. This leads to the so-called Fourier transformation. The Fourier transformed $\tilde{f}(\omega)$ of a not necessarily periodic function $f(t)$ is defined by

$$
\tilde{f}(\omega) \equiv \int_{-\infty}^{\infty} d t f(t) \cdot e^{-i \omega t}
$$

As usual $i^{2} \equiv-1$. There is also a backward transformation given by

$$
f(t)=\frac{1}{2 \pi} \int_{-\infty}^{\infty} d t \tilde{f}(\omega) \cdot e^{i \omega t}
$$

The proof of Eq. (3) or (4) is again performed by inserting Eq. (3) into Eq. (4) or vice versa. The Fourier transformed exists if the integral in Eq. (3) exists. Eq. (4) is the continuous analogue to Eq. (1). The sum in Eq. (1) is transformed into an integral and the discrete coefficients $a_{k}$ and $b_{k}$ are now a function $\tilde{f}(\omega)$. Please do not be confused that $\tilde{f}(\omega)$ has complex values (even if $f(t)$ is real). The identity

$$
e^{i \omega t}=\cos (\omega t)+i \cdot \operatorname{si}(\omega t)
$$

shows that the real part of $\tilde{f}(\omega)$ corresponds to $a_{k}$ and the imaginary part to $b_{k}$. In this sense one sometimes speaks of the cosine or sine transformed function. In the same token one may use Eq. (5) to rewrite Eq. (1) into

$$
f(t)=\sum_{k=-\infty}^{\infty} c_{k} \cdot e^{k \cdot i \omega t}
$$

with $c_{k} \in \mathbb{C}$ given by

$$
c_{k}=\frac{1}{T} \int_{0}^{T} d t f(t) \cdot e^{-k \cdot i \omega t}
$$

Applying it to share prices is now possible. The variation of stock prices can be described by a function $f(t)$. Eq. (2), (3), or (7) can be used to determine the frequency spectrum and build a ratio like:

$$
\frac{\sum_{k=0}^{K}\left|c_{k}\right|}{\sum_{k=0}^{\infty}\left|c_{k}\right|}
$$


With $\omega=2 \pi / T$ the sum in the numerator gives the sum of the (angular) frequencies starting from zero until $2 \pi K / T$. Of course, low frequencies belong to a longer time span. In the case of stock quotes, one may find it reasonable that price fluctuations adjust for new information within one day to three months, as being the frequency of publishing quarterly financial statements. Longer timespans until one year indicate irrationality as dividends and cash flows rather change as a smooth polynomial function.

The main mathematical problem with stock prices is that they are (strictly speaking) not a function of time in the sense required here. They are always mapping a finite number of time points on a finite number of prices. However, any integral over such functions is zero. This contrasts with discrete time series in natural science. There one may observe or calculate data at certain time points via digital or numerical approaches. However, the data does exist at any time. A function from $\mathbb{R} \rightarrow \mathbb{R}$ or even $\mathbb{C} \rightarrow \mathbb{C}$ can be obtained by interpolation.

Stock prices are men-made due to trading. They do not exist per se. Therefore, one may gather stock prices every second. Connecting them by straight lines appears to be a reasonable interpolation. However, it must be kept in mind that stock exchanges are predominantly closed for more than 10 hours in between trading days and around $30 \%$ of calendar days are no trading days. Additionally, having a stock price every second will never cover every price change on a tick basis. Furthermore, it is hardly possible to scrutinize reliable data covering every second especially from several stock exchanges. It is even challenging to get these data for a longer period of say the last 20 years. In this paper each stock price per day was generated by taking the mean of the daily open and close price. Beyond filtering for unreliable data even with cleaned data there is still an ambiguity at weekends and holidays. Therefore, the most reasonable approach with these data would be to connect the consecutive points by straight lines or even better exponential functions. Because of dealing with financial markets an interpretation of (positive or negative) compound interest seems to be appropriate. From that results a continuous function of time and Eq. (3) can be used to get the Fourier transformed. Of course, the backward transformation via Eq. (4) will lead to the exact stock price. The integral in Eq. (3) can be calculated analytically, because it is just an integral over a piecewise exponential function. However, this approach consumes vast amounts of computing power. Furthermore, it is doubtful whether it will lead to better results than given by the method described below. Though one should perform these calculations in the future, when faster data processing power will reach the threshold to reduce calculation time into a matter of days, in order to prove it.

A second approach would be to assume that the price of a stock is always constant over one trading day. This will lead to a rectangular curve. Because integrals can be taken over discontinuous functions Eq. (3) or (7) can be used to calculate the Fourier transformed or the amplitudes $c_{k}$ respectively. Please note that this will consume the same computing power as the exponential interpolation of the last paragraph. However, there is another problem. A rectangular curve changes within zero time at its discontinuous points. So, it will lead to infinite high frequencies, which are not connected to the stock price. The so found short frequencies will be high, even if there are none. For instance, consider a stock which grows constantly by five percent annually. Having just one price per day, the approach would lead to high frequencies, though a Fourier transformed of an exponential curve will decay with $1 / \omega$ for $\omega \rightarrow \infty$.

Therefore, the best approach to realize a fast and practicable process is via the discrete Fourier transform, even though the historical stock quotes are obviously neither weakly stationary nor equidistant. These shortcomings must be accounted for via detrending and the assumption 
that there is no difference if the market was opened or closed the day before. The mathematics behind the discrete Fourier transform is as follows. Instead of starting with $\omega=2 \pi / \mathrm{T}$ (with $\mathrm{T}$ in this paper being 20 years) which would lead to arbitrary high frequencies and an infinite number of $c_{k}$, one may choose a $T_{d} \approx \mathrm{T}$ with $k_{\max } / T_{d} \hat{=} 1$ day. This will reduce calculation time tremendously. Furthermore, it will be consistent to have a finite amount of amplitudes for a finite amount of data points. Squaring the time series as well as its frequency spectrum equals the same power on both sides known as the Parseval's theorem which leads to the power spectrum and shows how the variances are distributed over the frequencies. In the next chapter the transformation of the historical stock quotes of SAP SE from the time domain to the frequency domain will be demonstrated and afterwards the power spectra of various stock price series will be evaluated.

\section{RESULTS FOR STOCKMAKETS}

As described above, the discrete Fourier transformation has been applied to all stocks in various indices such as the Deutsche Boerse AG German Stock Index (DAX). A period of 20 years from 1997-01-02 until 2016-12-30 was considered, containing slightly over 5,000 trading days or data points for each stock. All raw data were derived from Thomson Reuters Eikon. Figure 1 shows the historical stock quotes of SAP SE in the time domain as well as its spectral counterpart with absolute values of $c_{k}$ in the frequency domain containing the equivalent information:
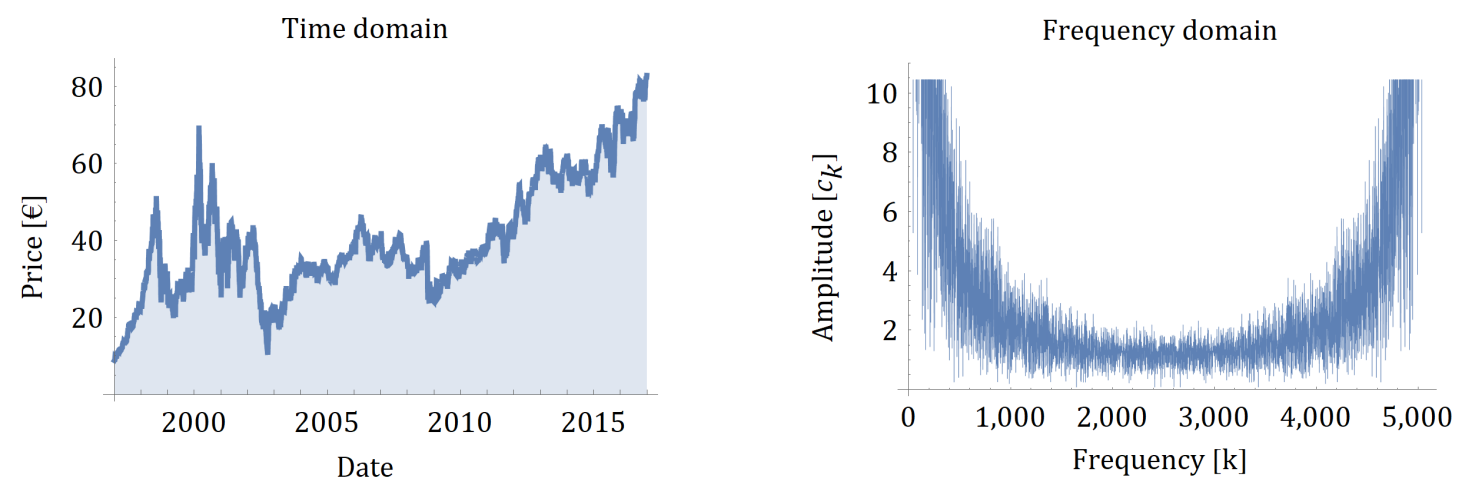

Figure 1. Time domain and Frequency domain of SAP SE - historical stock quotes

The absolute values of $c_{k}$ were taken. This also implies symmetry because the discrete Fourier transformation gives a complex amplitude and its conjugate. Furthermore, $a_{k}$ and $b_{k}$ of Eq. (2) may have positive or negative values while $\left|c_{k}\right|$ is always positive. Also, the real and imaginary part of $c_{k}$ can be used, which correspond to $a_{k}$ and $b_{k}$ of Eq. (2), respectively. The representation of the frequency domain in this form is for demonstration purposes only.

Several procedures have been applied to avoid undue disturbances. One important step was to extract the (exponential) growth or decay of the stock price. Essentially, the trend has been subtracted from the time series. It leads to a stock price fluctuating around zero. That leaves all relevant frequencies untouched but removes the trivial ones due to growth or decay.

The lowest frequency is given by $1 / 20$ years. This is artificial due to the period of 20 years under consideration. Because of the detrending, very low frequencies are reduced to a minimum. The high frequencies above 1/10 days are not reliable because of having just one price per day, so only frequencies from $1 / 10$ days until $1 / 1$ year were considered. The accumulated signal power in the range of three months to one year, defined as the sum squared amplitudes, in the numerator is divided by the power of the range from 10 days until 1 
year which results the irrationality of the stock. The power of the range from 10 days until three months is therefore seen as the inherent variance of the specific stock.

The irrationality of the DAX shares with uninterrupted data for the period under consideration is shown in Table 1:

Table 1. Irrationality of DAX members

\begin{tabular}{|lrr|}
\hline Company & Irrationality & Volatility \\
Muenchener Rueck AG & $73.7 \%$ & $26.7 \%$ \\
Beiersdorf AG & $76.6 \%$ & $22.8 \%$ \\
Bayer AG & $76.9 \%$ & $25.2 \%$ \\
E.ON SE & $77.2 \%$ & $25.0 \%$ \\
Siemens AG & $77.4 \%$ & $28.1 \%$ \\
Henkel AG \& Co KGaA & $77.5 \%$ & $21.9 \%$ \\
Adidas AG & $77.7 \%$ & $25.5 \%$ \\
BASF SE & $77.8 \%$ & $23.7 \%$ \\
RWE AG & $77.8 \%$ & $25.3 \%$ \\
Allianz SE & $77.9 \%$ & $29.2 \%$ \\
Linde AG & $78.2 \%$ & $22.5 \%$ \\
Fresenius Medical Care AG \& Co KGaA & $78.7 \%$ & $23.7 \%$ \\
Merck KGaA & $79.0 \%$ & $23.6 \%$ \\
Bayerische Motoren Werke AG & $79.1 \%$ & $28.7 \%$ \\
SAP SE & $79.2 \%$ & $32.5 \%$ \\
Volkswagen AG & $79.8 \%$ & $30.9 \%$ \\
Daimler AG & $80.1 \%$ & $28.4 \%$ \\
Continental AG & $80.3 \%$ & $30.8 \%$ \\
HeidelbergCement AG & $80.5 \%$ & $29.1 \%$ \\
Deutsche Lufthansa AG & $80.9 \%$ & $28.3 \%$ \\
Commerzbank AG & $82.2 \%$ & $36.8 \%$ \\
Deutsche Bank AG & $82.8 \%$ & $33.1 \%$ \\
Thyssenkrupp AG & $82.8 \%$ & $30.1 \%$ \\
Deutsche Telekom AG & $84.6 \%$ & $27.0 \%$ \\
\hline Mean & $79.1 \%$ & $27.4 \%$ \\
Median & $78.9 \%$ & $27.6 \%$ \\
\hline
\end{tabular}

On average the irrationality in the index was $79.1 \%$ for the stocks included with the median being slightly below the mean. A cluster of conservative titles like insurance and basic utilities forms the lower band of irrationality. The automotive industry begins above the mean and is very concentrated in the range from $79.1 \%$ to $80.3 \%$. The volatile and structurally risky companies Commerzbank AG, Deutsche Bank AG and Thyssenkrupp AG form the upper end of the ranking together with the Deutsche Telekom AG. The high irrationality of Deutsche Telekom AG is no surprise. After being privatized in 1996 right before the rise and fall of the Dotcom bubble it became one of the most notorious examples of irrational exuberance.

The difference between irrationality and volatility becomes apparent when comparing Muenchener Rueck AG and Deutsche Telekom AG, which have the lowest and highest irrationality within the evaluation. Muenchener Rueck AG has a volatility of $26.7 \%$ and thus a value slightly below the mean and median, although it has the lowest irrationality. The low value of irrationality results from the fact that the contribution of the irrational frequency range to the historical stock quotation is just 2.8 times higher than that of the rational range. Deutsche Telekom AG on the other side, while having a similar volatility, has a factor of 5.5. The 
higher factor results both from a lower contribution of the short frequencies to the overall share price development and from a higher contribution of the longer frequencies compared to the Muenchener Rueck AG.

In addition to the results above, an overview of irrationality ranges measured for selected indices is presented in Table 2 . The values refer to individual shares contained in the index, not to the index itself.

Table 2. Irrationality range of selected indices

\begin{tabular}{|lcccr|}
\hline Index & Min & Max & Mean & Median \\
S\&P 500 & $66.7 \%$ & $89.0 \%$ & $78.1 \%$ & $78.3 \%$ \\
FTSE & $68.3 \%$ & $84.3 \%$ & $77.1 \%$ & $77.7 \%$ \\
Nasdaq & $69.0 \%$ & $84.1 \%$ & $78.5 \%$ & $78.4 \%$ \\
Dow Jones & $72.7 \%$ & $80.4 \%$ & $77.0 \%$ & $77.0 \%$ \\
DAX & $73.4 \%$ & $84.6 \%$ & $79.1 \%$ & $78.9 \%$ \\
IBEX & $73.8 \%$ & $82.4 \%$ & $79.1 \%$ & $79.5 \%$ \\
HSI & $74.0 \%$ & $83.8 \%$ & $79.9 \%$ & $80.4 \%$ \\
\hline
\end{tabular}

Starting with the mean and median, both give a clear picture of the Dow Jones being the index with the lowest irrationality. The minimum (Min) and maximum (Max) of the range of irrationality behaves logically. The more companies included in the index, the wider the range of expected possible outcomes. Therefore, the similar ranges of irrationality for DAX, Spanish Exchange Index (IBEX) and Hang Seng Index (HIS) are plausible.

Please note, that as described above only qualifying stocks regarding data length and consistency are included. Further studies with shorter time periods and other data sources like from the Center for Research in Security Prices (CRSP) may include more companies.

Possible shortcomings and sources of errors lie in the fact that historical stock quotes are neither weakly stationary nor equidistant nor periodic. Different detrending methods will lead to slightly different results due to possible influence on the first frequency bins under one year. In this case, the values of $c_{k}$ in the low frequencies are reduced and thus the proportion of irrationality is lower than it would be. The ranking should nevertheless be stable, as the impact on each stock has a similar effect. Possible detrending methods are linear regression models e.g. via the ordinary least square method or one can apply a discrete wavelet-analysis as it can handle nonstationary time series, also low-pass filters are reasonable. Differencing of logarithmized time series is a very specific method of detrending which - even though being commonly applied in economics - cannot be applied here.

The point that weekends and holidays were ignored is probably a minor point for the time span under consideration. It will not add extra frequencies or eliminate others. Further studies with intraday data where non-equidistant data may impact the evaluation should consider the use of the Lomb-Scargle periodogram $[8,9]$.

Moreover, it should be noted that a reduction in measurement values necessarily leads to a broader frequency spectrum comparable to Heisenberg's "Uncertainty Principle" and vice versa [10]. Because of dealing with nonperiodic time series it is therefore important that the time period for the analysis is well above the longest frequency considered (in this analysis one year). 
In conjunction with the above-mentioned methods the concept of irrationality is transferable to other financial markets like bonds, housing prices or derivatives as well as to different time periods.

A more technical aspect are dividends. After a dividend has been paid, the stock price drops correspondingly. This rapid drop has an impact on the high frequencies in the frequency domain but only occurs at less than 1.6 percent of the trading days for quarterly dividendpaying companies.

\section{CONCLUSIONS}

The analysis shows that irrationality can classify stocks within an index according to risk and can be directly reconciled with historical volatility or beta analyses with minor transformations in representation. Therefore, the same restrictions as for volatility and beta apply. A single ratio cannot capture all risks at every point in time and must be accompanied by stress tests or scenario analyses including further information like liquidity or funding.

Additionally, the results show that there exists valuable information unobservable in the time domain with promising results for analyses within an index. Further studies should therefore compare irrationality to volatility and beta with respect to the compound annual growth rate (CAGR) to test the applicability and informative power of this risk measure. At least, the results are expected to show irrationality being somehow proportional to the other two risk measures. Furthermore, it should be investigated whether conclusions can be drawn about the instability of volatility by comparing long frequency ranges with short ones.

The concept of irrationality is transferable to other financial markets as for bonds, housing prices or derivatives as well as to different time periods.

\section{References}

Mandelbrot, B. The Variation of Certain Speculative Prices. The Journal of Business 1963, 36, 394-419.

Fama, E.F. Random Walks in Stock Market Prices. Financial Analysts Journal 1965, 21, 55-59.

. Efficient Capital Markets: A Review of Theory and Empirical Work. The Journal of Finance 1970, 25,

383-417, doi:10.2307/2325486.

Shiller, R.J. Irrational exuberance, Revised and expanded third edition; Princeton University Press: Princeton, 2016.

Summers, L.H. Does the Stock Market Rationally Reflect Fundamental Values? The Journal of Finance 1986, 41, 591-601, doi:10.2307/2328487.

Shiller, R.J. Do Stock Prices Move Too Much to Be Justified by Subsequent Changes in Dividends? American Economic Review 1981, 71, 421-436.

Appel, D.; Grabinski M. The origin of financial crisis: A wrong definition of value. Portuguese Journal of Quantitative Methods 2011, 3, 33-51.

Lomb, N.R. Least-squares frequency analysis of unequally spaced data. Astrophysics and Space Science 1976, 39, 447-462, doi:10.1007/BF00648343.

Scargle, J.D. Studies in astronomical time series analysis. II - Statistical aspects of spectral analysis of unevenly spaced data. Astrophysical Journal 1982, 263, 835-853.

Hill, M. The uncertainty principle for Fourier transforms on the real line. University of Chicago 2013. 